Network Working Group

Request for Comments: 3616

Category: Informational
F. Bellifemine

Telecom Italia Lab

I. Constantinescu

EPFL

S. Willmott

UPC

September 2003

\title{
A Uniform Resource Name (URN) Namespace for \\ Foundation for Intelligent Physical Agents (FIPA)
}

Status of this Memo

This memo provides information for the Internet community. It does not specify an Internet standard of any kind. Distribution of this memo is unlimited.

Copyright Notice

Copyright (C) The Internet Society (2003). All Rights Reserved.

Abstract

This document describes a Uniform Resource Name Namespace

Identification (URN NID) for the Foundation for Intelligent Physical Agents (FIPA). This URN NID will be used for identification of standard components published by the FIPA standards body in the area of Agent technology.

Table of Contents

1. Introduction. . . . . . . . . . . . . . . . . . . . . 2

2. Specification Template. . . . . . . . . . . . . . . . . 2

3. Namespace Considerations. . . . . . . . . . . . . . . . . . . 4

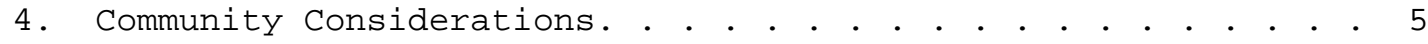

5. IANA Considerations . . . . . . . . . . . . . . . . . 6

6. Security Considerations . . . . . . . . . . . . . . . . 6

7. References. • . . . . . . . . . . . . . . . . . . . 6

7.1. Normative References. . . . . . . . . . . . . . 6

7.2. Informative References. . . . . . . . . . . . . . 6

8. Authors' Addresses. . . . . . . . . . . . . . . . . . . . . . 7

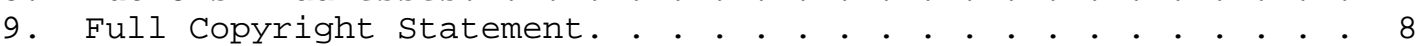




\section{Introduction}

The Foundation for Intelligent Physical Agents (FIPA) was established in 1996 in Geneva, Switzerland. FIPA is an international non-profit association of companies and organizations dedicated to promoting the industry of intelligent agents by openly developing specifications supporting interoperability among agents and agent-based applications. The core mission of the FIPA standards consortium is to facilitate the interworking of agents and agent systems across multiple vendors' platforms. Since its inception, FIPA has counted more than 60 member companies from over 20 different countries worldwide.

FIPA publishes technical specifications, schemas, diagrams and other documentation related to the area of Agent technology. FIPA wishes to create and manage globally unique, persistent, locationindependent identifiers for these resources.

2. Specification Template

Namespace ID:

"fipa"

Registration Information:

Version 1

Date: 2002-02-28

Declared registrant of the namespace:

Name: $\quad$ FIPA Secretariat (J. Kelly)

E-mail: secretariatefipa.org

Affiliation: Foundation for Intelligent Physical Agents (FIPA)

Address: $\quad \mathrm{C} / \mathrm{O}$ Me Jean-Pierre Jacquemoud

2 , rue Bellot

$\mathrm{CH}-1206$ Geneve

Declaration of structure:

The identifier has a hierarchical structure as follows:

urn: <assigned number>: $\{$ subspace-id1 $\}:\{$ subspace-id2 $\}: \ldots$

subspace-ids can be any US-ASCII string compliant with URI syntax and not containing the ":" character. The subspaces are used to divide the assigned namespace along one or more logical schemes. 


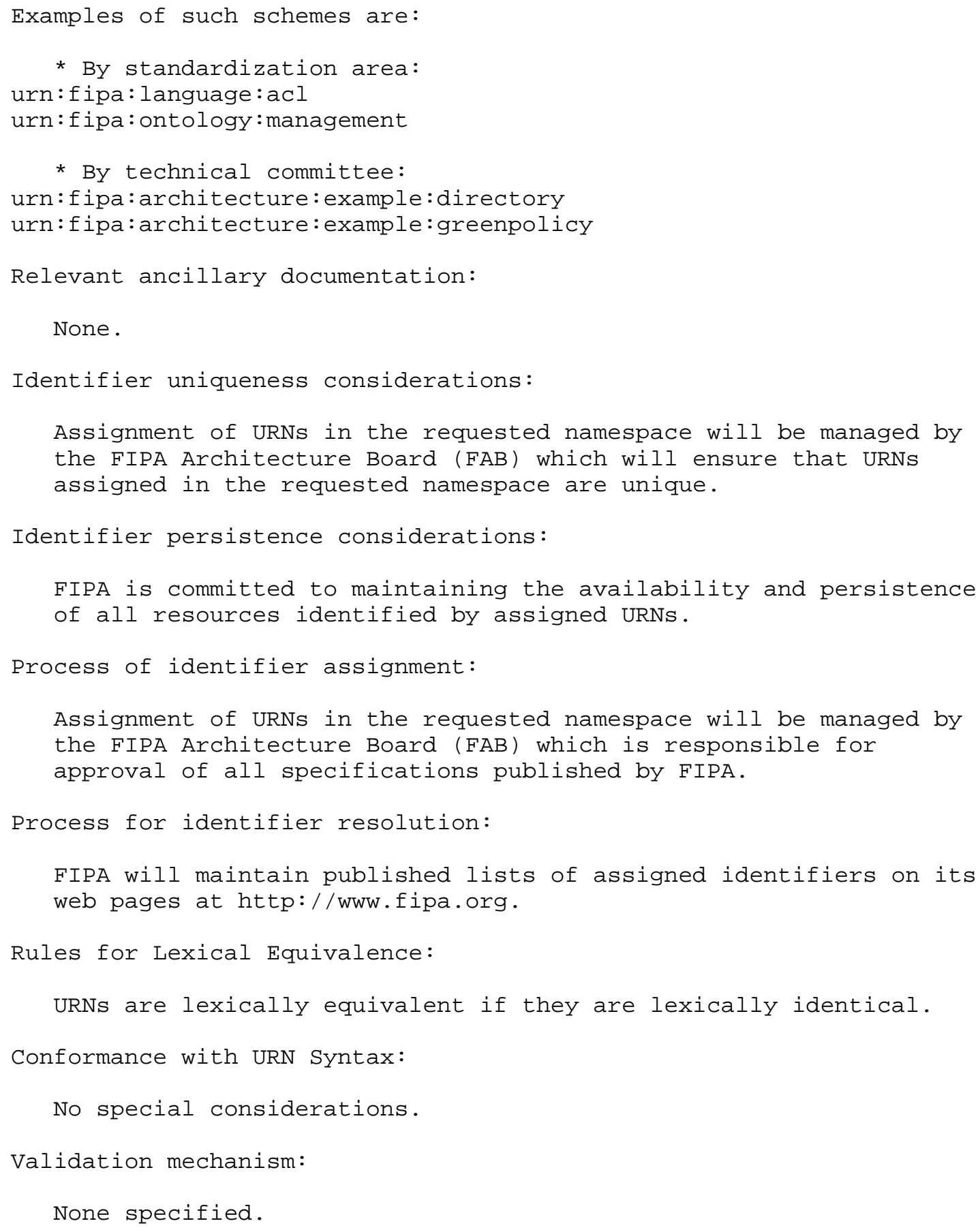


Scope:

Global.

3. Namespace Considerations

As part of the consideration of the URN namespace issue, FIPA has engaged in a survey of existing and proposed URN NID schemes which might be used instead of applying for a dedicated scheme. FIPA's specific requirements are that the namespace should be:

- Globally unique

- Persistent

- Location-independent

- Reflect the logical structure of FIPA specifications (see section 3)

- Be purely logical and without reference to "location" related elements such as (in particular) domain names/Domain Name System (DNS) entries such as URLs

- Be managed by the FIPA organisation to ensure the integrity, correctness and uniqueness of the namespace.

The closest to the requirements identified by FIPA were the proposed Vocabulary (VOC) [4] and TAG [3] schemes:

- In particular, TAG could be used in conjunction with the domain name "fipa.org" which FIPA has registered to generate unique identifiers. However, the problems with this scheme from FIPA's perspective are (in order of importance):

* It has not yet (to our knowledge) been approved / accepted by IESG or IANA (making it inappropriate for use in a standards document).

* The labels generated related to the time date, owner of the label but specify no particular semantics for the content/text of the label which would be desirable to identify a hierarchy of standards.

* The uniqueness in the scheme is DNS based.

- VOC provides a more logical structure for a logical hierarchy of specifications but still presents the following problems:

* It has not yet (to our knowledge) been approved / accepted by IESG or IANA making it inappropriate for use in a standards document). 
* The uniqueness in the scheme is DNS based.

In both cases, there is no clear authority for managing the correctness of the namespace.

4. Community Considerations

FIPA standards address the area of interoperability between autonomous software systems (agents) in open environments such as company intranets or the Internet itself. Communication between such systems is highly contingent on developers and the systems themselves being able to unambiguously identify which technology specifications (interaction protocols, languages, domain descriptions and the like) are to be used for particular purposes in any given interaction.

The use of a managed, unique namespace for FIPA specification components would therefore be of great benefit:

- To the FIPA standards process: providing unique labels for individual specification components.

- To developers implementing systems based on FIPA standards: identifying which specifications are in use in a particular system, allowing them to develop software able to flexibly distinguish between different technologies used.

- To users of networks making use of FIPA technology (in whole or in part): improving the integrity and clarity of interactions in the system, allowing them to precisely identify technology requirements for interacting with any given software system using FIPA technology.

Whilst the assignment of identifiers is managed by the FIPA Architecture Board the application for identifiers is through the open FIPA standards process - thus anybody following the process is entitled to request an identifier for technologies they consider relevant to FIPA's work.

Final acceptance of any FIPA standard is contingent on a vote of the FIPA membership. Although membership is not free (see [2] for details of costs), it is open to any organisation with an interest in FIPA's work.

The initial plans for identifier resolution are to host a web page on http://www.fipa.org to provide for resolution of identifiers FIPA will also encourage third parties (FIPA members and others) to host 
more advance dereferencing service or to develop software to provide dereferencing where these provide a correct and accurate reflection of the namespace. FIPA itself may also engage in such activities.

5. IANA Considerations

The IANA has registered formal URN namespace 14, to FIPA within the IANA registry of URN NIDs.

6. Security Considerations

The FIPA URN Namespace ID shares the security considerations outlined in RFC 3406 [1], but has no other known security considerations.

7. References

7.1. Normative References

[1] Daigle, L., van Gulik, D., Iannella, R. and P. Faltstrom, "Uniform Resource Names (URN) Namespace Definition Mechanisms", BCP 66, RFC 3406, October 2002 .

7.2. Informative References

[2] Foundation for Intelligent Physical Agents. http://www.fipa.org/

[3] Kindberg, T. and S. Hawke, "The 'tag' URI scheme and URN namespace", Work in Progress, September 2001.

[4] Stickler, P., "The 'voc:' URI scheme for Vocabulary Terms and Codes, Work in Progress, January 2002. 
8. Authors' Addresses

Fabio Bellifemine

Chair of the FIPA Architecture Board

Telecom Italia Lab

EMail: Fabio.BellifeminedTILAB.COM

Ion Constantinescu

EPFL

EMail: ion.constantinescudepfl.ch

Steven Willmott

Universitat Polit/210cnica de Catalunya

EMail: stevedsi.upc.es 
9. Full Copyright statement

Copyright (C) The Internet Society (2003). All Rights Reserved.

This document and translations of it may be copied and furnished to others, and derivative works that comment on or otherwise explain it or assist in its implementation may be prepared, copied, published and distributed, in whole or in part, without restriction of any kind, provided that the above copyright notice and this paragraph are included on all such copies and derivative works. However, this document itself may not be modified in any way, such as by removing the copyright notice or references to the Internet society or other Internet organizations, except as needed for the purpose of developing Internet standards in which case the procedures for copyrights defined in the Internet Standards process must be followed, or as required to translate it into languages other than English.

The limited permissions granted above are perpetual and will not be revoked by the Internet society or its successors or assignees.

This document and the information contained herein is provided on an "AS IS" basis and THE INTERNET SOCIETY AND THE INTERNET ENGINEERING TASK FORCE DISCLAIMS ALL WARRANTIES, EXPRESS OR IMPLIED, INCLUDING BUT NOT LIMITED TO ANY WARRANTY THAT THE USE OF THE INFORMATION HEREIN WILL NOT INFRINGE ANY RIGHTS OR ANY IMPLIED WARRANTIES OF MERCHANTABILITY OR FITNESS FOR A PARTICULAR PURPOSE.

Acknowledgement

Funding for the RFC Editor function is currently provided by the Internet society. 